\title{
Editorial
}

\section{A human mitochondriopathy caused by AIF mutation}

\author{
N Modjtahedi ${ }^{\star, 1,2,3}$, F Giordanetto ${ }^{4}$ and G Kroemer ${ }^{\star, 1,2,3}$ \\ Cell Death and Differentiation (2010) 17, 1525-1528; doi:10.1038/cdd.2010.88
}

Mitochondrial diseases, also called 'mitochondriopathies', include a large number of heterogeneous, systemic or organ-specific diseases that primarily affect infants and are explained by an inborn or progressive dysfunction of mitochondrial oxidative phosphorylation. ${ }^{1}$ These genetically transmitted diseases have an incidence lower than 1:10000 and are explained by mutations in either mitochondrial or nuclear DNA-encoded genes that are indispensable for the structure, function or regulation of the five multiprotein complexes that compose the respiratory chain (RC). ${ }^{1}$ Many among the nuclear mutations responsible for mitochondriopathies have been identified, but others have not yet, in spite of the fact that their precise identification is pivotal for molecular diagnostics, genetic counseling, cognitive insights into the molecular pathogenesis of the disease, as well as the rational design of therapeutic strategies. Now, a fascinating paper by Ghezzi et al. ${ }^{2}$ associates several cases of X-linked progressive mitochondrial encephalomyopathies with a specific mutation in the AIFM1 gene, which encodes a protein that is well familiar to the cell death community: apoptosisinducing factor (AIF). ${ }^{3}$ In a certain sense, the work by Ghezzi et $a .^{2}$ consecrates over 15 years of dedicated work on the function of AIF, which has previously been characterized in multiple model organisms including yeast, flies, nematodes and mice. ${ }^{3}$ Finally, after all these years, a human disease shows the pathophysiological relevance of AIF.

The AIFM1 gene, which is localized on human chromosome $X$ (Xq25-Xq26), encodes the 613-amino acid AIF precursor that carries an $\mathrm{N}$-terminal mitochondrial localization signal. ${ }^{3}$ In healthy cells, once the precursor protein is translated in the cytoplasm and imported into mitochondria, the mitochondrial localization signal is cleaved by a mitochondrial peptidase and the mature AIF protein is inserted into the inner membrane, facing the intermembrane space. ${ }^{3}$ AIF owes its name to its initial characterization as the first caspase-independent death effector that induces chromatin condensation and DNA fragmentation when added to isolated nuclei in vitro. ${ }^{3}$ Indeed, upon mitochondrial outer membrane permeabilization, which constitutes the rate-limiting step of most (if not all) apoptotic pathways, ${ }^{4}$ AIF is released from mitochondria and translocates first to the cytosol and then to the nucleus, where it contributes to apoptotic chromatin condensation and DNA degradation. $^{3}$
Today, it is well established that AIF is not a universal cell death effector and that its variable contribution to the execution of cell death depends on the cell type and on the apoptotic insult. ${ }^{3}$ Thus, AIF lethal activity is considered to have a crucial role in the programmed death of neurons and photoreceptor cells provoked by excitotoxins, hypoxiaischemia, hypoglycemia or deprivation from trophic support, as in the case of retinal detachment. ${ }^{3}$ The apoptotic release of the mitochondrial membrane-anchored AIF relies on its $\mathrm{N}$-terminal cleavage by calcium-dependent cysteine proteases of the calpain or cathepsin families, which renders the protein soluble. ${ }^{3}$ AIF cleavage is often secondary to the hyperactivation of PARP1, a nuclear DNA repair enzyme involved in the DNA damage response, as this occurs for instance in dying neurons in response to ischemia or excitotoxicity. ${ }^{3,5}$ In this context, AIF is either released as a consequence of calpain activation or the accumulation of poly(ADP-ribose) polymers generated by PARP1. ${ }^{3}$ It is controversial whether mitochondrial outer membrane permeabilization is always required for AIF release or a poorly characterized pool of AIF tethered to the surface of the outer mitochondrial membrane ${ }^{6}$ may be released in a mitochondrial outer membrane permeabilization-independent manner.

Irrespective of these details, AIF is more than just a latent killer that, once liberated from restraint, sneaks into the nucleus to kill the cell. Indeed, AIF has an essential vital role in normal bioenergetic metabolism, similar to other bifunctional proteins of the mitochondrial intermembrane space, such as cytochrome $c .^{7}$ A large part of the C-terminus of AIF has a similar structure as bacterial nicotinamide adenine dinucleotide-dependent oxidoreductases, and recombinant AIF protein can indeed oxidize NADH in vitro. ${ }^{3}$ Upon its import into the mitochondrion, AIF incorporates its cofactor flavin adenine dinucleotide (FAD), a step that likewise is required for proper AIF folding and stabilization. The two FAD-binding segments (residues 128-262 and 401-480), as well as the NADH binding domain (residues 263-400) of human AIF, are highly conserved throughout phylogeny (AIF orthologs have been identified in Saccharomyces cerevisiae, Caenorhabditis elegans, and Drosophila melanogaster), as well as among the members of the AIF gene family (there are additional AIF homologues conserved during the evolution). ${ }^{3}$ So far, the enzymatic reaction that is catalyzed by AIF within

\footnotetext{
${ }^{1}$ INSERM U848, Villejuif, France; ${ }^{2}$ Institut Gustave Roussy, Villejuif, France; ${ }^{3}$ University Paris 11, Orsay, France and ${ }^{4}$ Lead Generation, Medicinal Chemistry, AstraZeneca R\&D Mölndal, Pepparedsleden, Mölndal, Sweden

*Corresponding authors: G Kroemer, INSERM, U848, Institut Gustave Roussy, Pavillon de Recherche 1, Villejuif, Paris F-94805, France.

Tel: + 331421160 46; Fax: + 331421160 47; E-mail: kroemer@ orange.fr or

N Modjtahedi, Tel: + 331421154 91; Fax: + 331421166 65; E-mail: nazanine@igr.fr
} 
mitochondria is enigmatic. However, the existence of a stable NADH-associated AIF dimer and the redox-dependent modification of its conformation suggest a tight control of AIF enzymatic activity through redox-dependent monomer-dimer transitions. $^{3}$

Attempts to create AIF null mice by homologous recombination have been unsuccessful because AIF's mitochondrial function is indispensible for cell survival during either early or advanced embryogenesis, depending on the knockout strategy and the genetic background. ${ }^{3}$ The specific deletion of Aif in the prospective midbrain and cerebellum revealed that AIF is necessary for cell-type specific neurogenesis. ${ }^{3}$ Accordingly, a defective cortical development and reduced neuronal survival was observed in mutant mice that specifically lost Aif in the telencephalon. ${ }^{3}$ Mutant mice with muscle-specific loss of AIF developed severe skeletal muscle atrophy and dilated cardiomyopathy before becoming lethargic around the age of 5 months. ${ }^{3}$ Mice lacking AIF specifically in hepatocytes had no major phenotype, although they tended to manifest a reduction in body weight and were resistant against high-fat diet-induced diabetes and obesity. ${ }^{3}$ In all organs from which AIF was removed by targeted knockout, mitochondrial respiration was compromised. This was accompanied by a reduction in the relative abundance of the protein subunits of complex I, which was the most affected one, although the function and abundance of complexes III, IV and V were also reduced in Aif-deficient embryonic stem cells, as well as in skeletal muscle or liver cells from which Aif has been removed by conditional knockout. ${ }^{3}$

These results have been confirmed by studying mice bearing the so-called Harlequin $(\mathrm{Hq})$ mutation (Figure 1a), in which a retroviral insertion in the first intron of Aif causes an $80 \%$ drop in the expression level of AIF. ${ }^{8}$ This hypomorphic Aif mutation is tolerable and has no major effect on mouse development yet impacts severely on the aging organism. ${ }^{3}$ $\mathrm{Hq}$ mice develop progressive ataxia and blindness due to the progressive loss of terminally differentiated cerebellar and retinal neurons. Moreover, signs of severe oxidative stress and degenerating mitochondria were detected in the brain of $\mathrm{Hq}$ mice. ${ }^{3}$ Complex I dysfunction was revealed in the degenerating $\mathrm{Hq}$ tissues (retina, cerebellum, thalamus, cortex, optical nerves), less so in the skeletal muscle and not at all in other organs (heart, liver, testis), ${ }^{3}$ unraveling a hitherto unexplained organ-dependent heterogeneity in the manifestation of genetically determined mitochondrial defects. The correlation between the downregulation of AIF in the degenerating $\mathrm{Hq}$ organs, the aggravation of complex I dysfunction and the clinical evolution of the Hq phenotype is intriguing, making $\mathrm{Hq}$ mice a valuable tissue-specific model of complex I deficiency syndrome. ${ }^{3}$ It has to be noted that complex I deficiency syndromes comprise almost $30 \%$ of human mitochondriopathies, ${ }^{1}$ and that many among these complex I deficiency syndromes have not been fully explored in genetic terms. In a collaborative study, the AIF genes of some 90 patients with idiopathic complex I deficiency syndromes were sequenced, without success. ${ }^{3}$ Thus, we failed to validate our postulate that some human complex I deficiencies might be due to AIF mutations when testing this hypothesis on a non-selected cohort of patients with complex I-specific mitochondriopathies.
When exploring two male infant patients born from monozygotic twin sisters with a common maternal grandmother (remember that the gene coding for AIF is located on the $X$ chromosome), Ghezzi et al. ${ }^{2}$ finally succeeded in attributing a severe early-onset progressive mitochondrial encephalomyopathy to a mutation in the AIF gene (Figure 1a). The pathogenic AIF mutation, defined by Ghezzi et al. ${ }^{2}$ consists the deletion of an entire triplet coding for arginine residue 201 (R201A) of the AIF precursor protein. The expression of the disease-associated allele impacts on the optimal function of oxidative phosphorylation but most importantly differences with respect to the AIF downregulation in the $\mathrm{Hq}$ mouse model are revealed (Figure 1a). Indeed, contrary to brain and retina cells from $\mathrm{Hq}$ mice, which revealed a preponderant $\mathrm{Cl}$ deficiency, ${ }^{3}$ fibroblasts from both AIF R201s patients exhibited an important CIII and CIV but mild C1 deficiency. ${ }^{2}$ The observed respiratory defects were the direct conse-

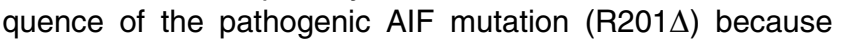
they were partially corrected by transfection-enforced overexpression of wild-type (wt) AIF or by continuous culture of AIF R201s fibroblasts in the presence of riboflavin, the precursor of $F A D$, which constitutes the prosthetic group of AIF. ${ }^{2}$ The multi-complex $(\mathrm{Cl}, \mathrm{CIII}$ and $\mathrm{CIV})$ dysfunction of $\mathrm{RC}$ activity that was detected in muscle biopsies could be correlated with a severe loss of mitochondrial DNA. ${ }^{2}$ Beyond putative species differences, it is extremely difficult to compare systematic studies on mouse models of reduced or absent AIF expression with isolated observations on samples from AIF R201 $\Delta$ patients, which express normal levels of an abnormal AIF protein. For this reason, it is urgent to generate knock-in mice in which the normal AIF protein has been replaced by one in which the R200 (R201 in humans) has been selectively deleted. This would facilitate the characterization of all $\mathrm{RC}$ complexes in multiple distinct organs.

The crystal and NMR structures of AIF $^{3}$ predict that R201, the arginine residue that is deleted in the novel pathogenic AIF mutant $\mathrm{R} 201 \Delta,{ }^{2}$ is part of the $\mathrm{N}$-terminal FAD binding domain of AIF. As such, it likewise has a crucial role in the accessibility of the FAD-binding active site and the spatial orientation of the functionally important residue W196, through its redoxdependent interaction with a regulatory loop located between residues 510 and $560 .^{2}$ Molecular modeling of the R201A mutant and in vitro experiments realized by Ghezzi et al. ${ }^{2}$ indicate that AIF R201 $\Delta$ mutant is unstable and possesses altered enzymatic properties compared with the wt protein. As the molecular basis for the mitochondrial activity of AIF is not understood, so far it is not clear whether R201ه affects the binding of AIF to nicotinamide adenine dinucleotide (or other yet unknown substrates), whether this mutation might affect the substrate specificity or whether it rather might affect interactions of AIF with other mitochondrial proteins. In other words, the present state of knowledge on AIF is rather fragmentary, and the identification of its preferential substrates (electron donors and acceptors), as well as that of its mitochondrial interactors, which may well be organized as 'metabolomes' is urgently awaited. As there is no indication for a physical interaction of AIF with specific protein subunits from RC complexes, ${ }^{3}$ it is elusive whether AIF is required for an optimal biogenesis/assembly of $\mathrm{RC}$ complexes, or whether AIF participates in the maintenance/repair of these 
A
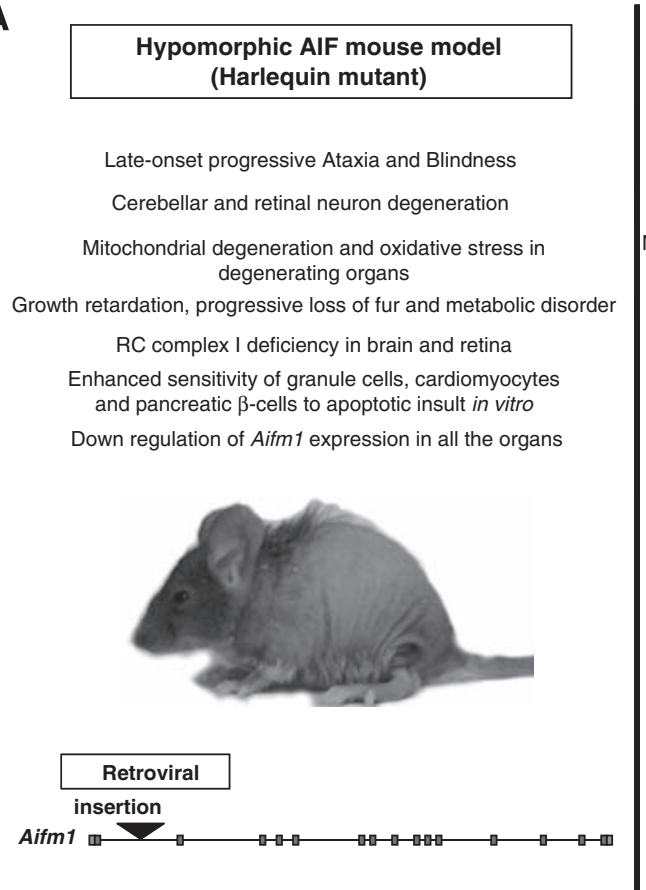

\begin{tabular}{|c|}
\hline $\begin{array}{c}\text { Pathogenic AIF mutation in } \\
\text { humans }\end{array}$ \\
\hline
\end{tabular}

Early-onset progressive mitochondrial encephalomyopathy Neurological and psychomotor development abnormalities Muscular atrophy, mitochondrial DNA loss and increased cell death in the muscle

\section{RC complex III and IV deficiency in fibroblasts and multi-} complex deficiency in muscle

Enhanced sensitivity of fibroblasts to apoptotic insult in vitro Disease-seggregating mutation in AIFM1
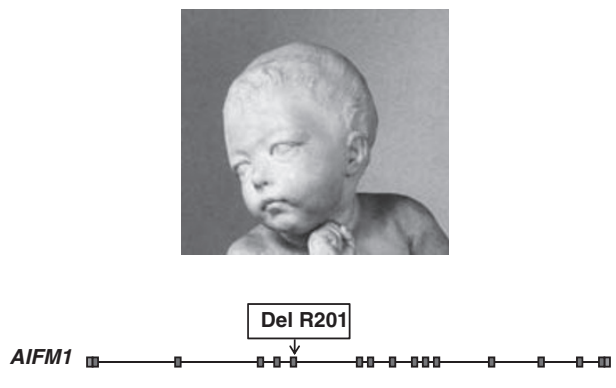

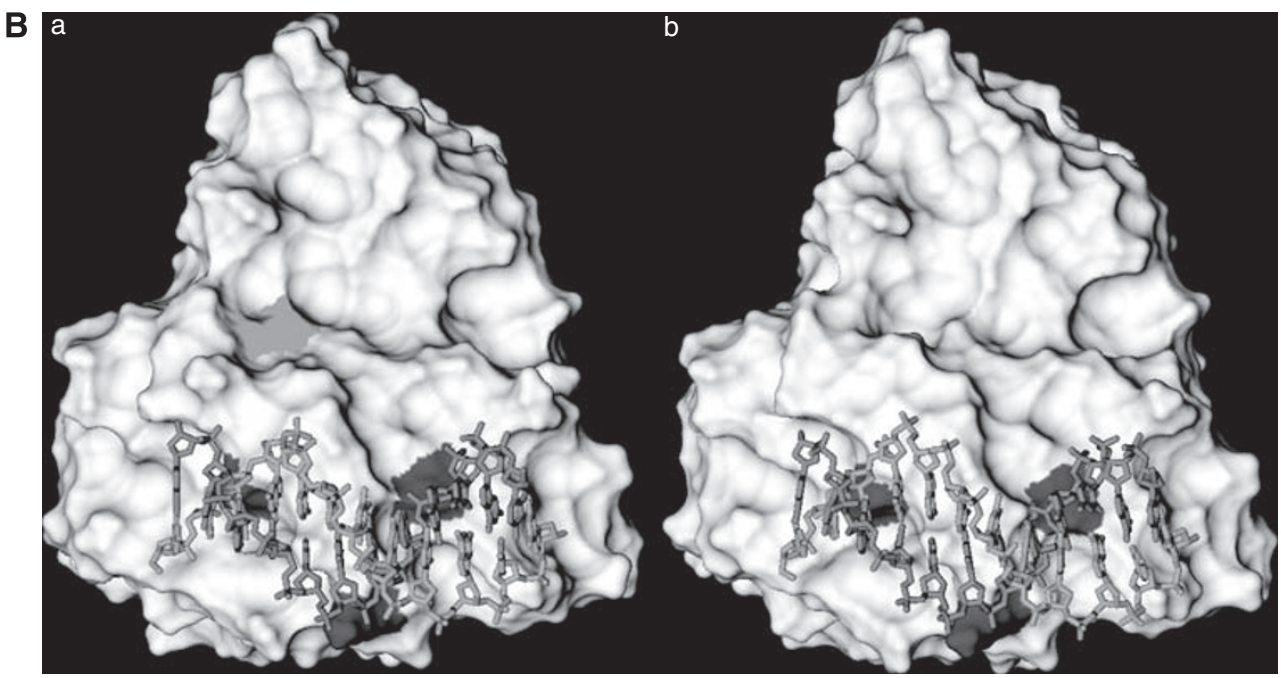

Figure 1 (A) Phenotypic comparison of AIF hypomorphic Hq mouse model with patients suffering from AIF-associated encephalomyopathy. Major phenotypic traits characterizing the AIF hypomorphic mutant $\mathrm{Hq}$ mouse model (left panel) and of patients carrying a pathogenic mutation of AIF (right panel) are listed. Schematic presentations of the murine Aifm1 and human AIFM1 genes are depicted; untranslated and translated exons are, respectively, colored in black and red. The retroviral insertion in the Aifm 1 (black triangle) is mapped to the first intron and the deletion of the residue Arg201 to the fifth exon of AIFM1 (black Arrow). RC. Citations are listed in the main text. Hq mouse photo is the courtesy of Klas Bloomgren. (B) Molecular modeling of the interaction between AIF and DNA. Predicted interaction between a 12 bp DNA duplex (orange and pink sticks) and (a) wt AIF (wt); (b) AIF (R201 $)$ ). The accessible surface of AIF is shown in white, highlighting the location of R201 in green and the main DNA-interacting partners K510, K518 and R584 in blue from left to right (numbering according to wt AIF). After rigid body docking of 12-mer DNA (PDB: 1fq2) to wt human AIF (PDB: 1m6i) and a modeled human AIF mutant lacking R201, the two systems were energy minimized, solvated, equilibrated and subjected to molecular dynamics simulations in a NPT ensemble for up to $5 \mathrm{~ns}$ using AMBER. The average minimized structures from such calculations are depicted here

multi-protein complexes. ${ }^{3}$ AIF might also be implicated in the regulation of the inner mitochondrial membrane structure and/ or stability because fragmented mitochondria with aberrant cristae were observed in cortical neurons of mutant mice that specifically lost Aif in the telencephalon. ${ }^{9}$ We recently described a brain-specific isoform of AIF (AIF2) that is produced through the alternative usage of exon 2 and differs from the ubiquitously expressed AIF (AIF1) only within its transmembrane region. ${ }^{10}$ Both AIF isoforms are localized in the same mitochondrial sub-compartment, can dimerize and are similar in their capacity to sustain a normal function of the $\mathrm{RC}$, but differ in their membrane anchorage capacity. ${ }^{10}$ Moreover, overexpression of the two AIF isoforms differentially affected the ultrastructure of mitochondria, suggesting 
that AIF, and in particular the transmembrane region of AIF, might affect the shape or stability of the mitochondrial inner membrane. ${ }^{10}$ Ghezzi et $a l^{2}$ have shown that cell culture in galactose- (rather than glucose-) containing media, a manipulation that forces cells to rely on mitochondrial respiration, provoked a much stronger mitochondrial fragmentation in fibroblasts expressing AIF R201 $\Delta$ than in control cell cultures expressing normal AIF, pointing again towards a yet-to-bedefined effect of AIF on mitochondrial dynamics. Moreover, upon culture in galactose, AIF R201s cells manifested an aggravated deficiency in $\mathrm{Cl}$, CIII and CIV activities. ${ }^{2}$ The cause-effect relationship between this exacerbated mitochondrial deficiency and mitochondrial fragmentation has yet to be determined.

AIF can directly bind to nucleic acids including singlestranded and double-stranded DNA. ${ }^{3}$ Crystal structures of AIF and mutagenesis experiments revealed the presence of positively charged amino acids that are scattered on the surface of the molecule and that are required for the interaction of recombinant AIF with DNA, as well as for the induction of nuclear apoptosis by overexpressed AIF. ${ }^{3}$ Ghezzi et al. $^{2}$ reported that the deletion of arginine 201 enhanced the DNA-binding capacity of mutant AIF (R201 $\Delta$ ) and rendered cells more sensitive to lethal stimuli. Accordingly, patients carrying the AIF R201 $\Delta$ mutation had an abnormally high percentage of TUNEL ${ }^{+}$cells in their muscles. ${ }^{2}$ Whether the nuclei of such cells (which are negative for active caspase-3) contain AIF, has not yet been determined though. Intrigued by the fact that R201 and the major DNA docking sites (K510, K518 and R584) on AIF are located at a large distance from each other, we modeled the molecular interaction between, on one hand, DNA and on the other hand wt versus AIF R201s (Figure 1b). After system equilibration, molecular dynamics simulations of the two complexes highlight a more stable AIF R201 $\Delta$-DNA complex and a stronger DNA-K510 interaction in AIF R2014 as compared with wt AIF (92 versus $69 \%$ occupancy, respectively). It is interesting that $\mathrm{K} 510$ is proximal to W196 ( $\alpha$-carbon distance $\sim 5 \AA$ ) and the perturbing effect of the R201 deletion on the 191-203 $\beta$-hairpin might indirectly affect K510's mobility and ability to interact with the phosphate backbone of the DNA duplex. The enhanced binding of mutant AIF (R201A) to naked DNA suggests a gain-of-function phenotype with respect to its lethal activity. Nonetheless, it will be important to determine whether mutant AIF (R201 $\Delta$ ) truly possesses an increased chromatin-condensing and DNA-degrading effect, because this activity does not only depend on the binding of AIF to DNA but also involves interactions between AIF and other proteins, in particular cyclophilin $A^{3,11}$ and histone $\mathrm{H} 2 \mathrm{AX} .^{11}$

In conclusion, the paper by Ghezzi et al. ${ }^{2}$ makes a milestone contribution to the genetic and functional exploration of human mitochondriopathies. Future work has to resolve whether other pathogenic mutations in the AIF gene exist and through which exact molecular pathways pathogenic AIF mutations, including the R201s mutation, can lead to respiratory deficiencies and cell death-related phenotypes. Ghezzi et al. ${ }^{2}$ show that supplementation with riboflavin can partially correct the respiratory deficiency of fibroblasts carrying the R201s mutation in vitro. Moreover, oral supplementation with riboflavin ameliorated the clinical status of one patient carrying the mutated AIF gene. Riboflavin can be administered at high doses without major toxic side effects to children with mitochondriopathies, ${ }^{12}$ and hence should be explored in more detail both at the clinical level and in preclinical models, for instance in mice carrying suitable AIF knock-in mutations.

\section{Conflict of interest}

The authors declare no conflict of interest.

Acknowledgements. The authors' own work is sponsored by INSERM and Ligue Nationale contre le Cancer (équipe labellisée).

1. Koene S, Smeitink J. J Intern Med 2009; 265: 193-209.

2. Ghezzi D et al. The American Journal of Human Genetics 2010; 86: 1-11.

3. Hangen E et al. Trends Biochem Sci 2010; 35: 278-287.

4. Green DR, Kroemer G. Science 2004; 305: 626-629.

5. Schreiber $V$ et al. Nat Rev Mol Cell Biol 2006; 7: 517-528.

6. Yu SW et al. ASN Neuro 2009; 1: e00021.

7. Gogvadze V, Orrenius S, Zhivotovsky B. Biochim Biophys Acta 2006; 1757: 639-647.

8. Klein JA et al. Nature 2002; 419: 367-374.

9. Cheung EC et al. EMBO J 2006; 25: 4061-4073.

10. Hangen E et al. Cell Death Differ 2010; 17: 1155-1166.

11. Artus $C$ et al. EMBO J 2010; 29: 1585-1599.

12. Bugiani $\mathrm{M}$ et al. Brain Dev 2006; 28: 576-581. 